\title{
Grid-Based GA Path Planning with Improved Cost Function for an Over-actuated Hover-Capable AUV
}

\author{
Kantapon Tanakitkorn*, Philip A. Wilson ${ }^{\dagger}$, Stephen R. Turnock ${ }^{\ddagger}$, and Alexander B. Phillips ${ }^{\S}$ \\ Fluid Structure Interactions Research Group, University of Southampton, UK \\ Email: *kt1e11@ soton.ac.uk, †Philip.Wilson@soton.ac.uk, ${ }^{\dagger}$ S.R.Turnock@ soton.ac.uk, ${ }^{\S}$ abp@ soton.ac.uk
}

\begin{abstract}
For an AUV to perform a long-range mission with its maximum endurance, its energy consumption during transit must be kept to a minimum. This paper presents an improved cost function for a grid-based genetic algorithm (GA) path planning in 2D static environments. The proposed function consists of energy consumption terms that are estimated according to dynamics of a hover-capable AUV - notably Delphin2 AUV. It seeks for a path that requires least effort for the vehicle to move along. A simulation was written in Matlab and the outcomes of the GA with the improved cost function are compared with the ones of a GA with an optimal distance approach as well as an $\mathrm{A} *$ approach. It is found that outcomes of an improved cost function require less energy compared with the other techniques.
\end{abstract}

Keywords-Autonomous Underwater Vehicle, AUV, Delphin2, Genetic Algorithm, Path Planning.

\section{INTRODUCTION}

Path planning is one of the most important pieces of software that is integrated in autonomous systems. It plays a role in finding a best valid path from a start location to a desired destination. There have been a number of path planning techniques proposed in the past decades, however, none of them are perfect. Each technique has been developed to suit a particular form of environment representation, hence, having their own strengths and limitations. Thus, the development of path planning is still an active area in robotic navigation.

Artificial Potential Field (APF) is a well known technique that adopt a concept of magnetic poles [1]. This causes a mechanism that the robot is pulled towards the goal and repelled from obstacles. A valid path is found by following a vector-sum of these forces. This technique can easily be extended to a higher dimension space with a little increase in computational effort. The main concern is it may fail to find even one solution as it is very sensitive to local minimum traps caused by concave-shaped obstacles or stagnation points [2]. Many attempts have been made to propose a local-minimum free APF [3], [4], [5], [6], however, the local minimum issue still remains.

$\mathrm{A}^{*}$ [7] and its variants [8], [9], [10], [11], [12] is one of the simple but practical pathfinders. A heuristic approach is used to make the exploration focus towards the goal compared to other graph search techniques such as Dijkstra's algorithm
[13]. This technique is very reliable as it guarantees a solution (if the solution does exist). A shortcoming is that it may only yield a sub-optimal solution as the path is constrained with 8-connectivity links [10].

The genetic algorithm (GA) is a stochastic search technique that was inspired from Dawins theory of evolution based on the principle of survival of the fittest [14], [15]. It has successfully been used in solving many optimization problems, including a path planning. This technique appears to produce a much smoother path compared with a classical search algorithm such as $\mathrm{A}^{*}$. In addition, GA is a global optimizer which is very unlikely to get trapped in a local minimum.

In this paper, a GA pathfinder with an improved fitness function for an overactuated AUVs is presented. The vehicle is assumed to travel at a constant depth and constant forward speed in a 2D static known environment. It is a fact that there is a finite energy stored on-board. To maximise the endurance of the AUV, it is required the most energy efficient path for the vehicle to move along. As such, the proposed cost function comprises of three terms: hotel load, propulsion load and manoeuvring load. With this, a solution of GA will be a best compromise between a shortest path which consumes least hotel and propulsion energy and a smoothest path which consumes least manoeuvring energy at the possible expense of a greater distance travelled.

This paper is structured as follows. Section II is related works. A brief introduction of Delphin2 AUV is provided in section III. Section IV explains the construction of a 2D grid map. Overview of the GA procedure is presented in section V. Parameters of path and improved cost function are discussed in section VI and VII. An experiment setup and results of the proposed algorithm are provided in section VIII and IX. Section $\mathrm{X}$ is a conclusion and future work.

\section{RELATED WORKS}

A GA path planner with a grid-based environment was first introduced in [16]. A fix-length binary chromosome was used. This constrains a path to $x$-, or $y$-monotone. Later, the orderly numbered grids technique was used to represent a working space [17], [18], [19]. This leads to the use of a variable-length chromosome coding scheme that significantly 
improves a performance and adaptivity of GA technique. Improved GA operators that suit the new chromosome coding scheme were presented in [17]. A variable-length chromosome representation that uses real $(x, y)$ coordinate was found in [20]. Despite all these improvements, most approaches evaluate a fitness of path based on a travelling distance.

There has been previous attempts made to improve the cost function of GA based path planners. Difficulties of navigating in hazardous zones were studied by [21], [22], [23]. For example, transiting through hazardous cells would require more effort than a normal zone. Each cell is classified and a cost of transit through hazards is assigned accordingly. Fitness functions that minimize the energy cost for a vehicle operating in ocean currents were proposed in [24] and [25]. The idea is to have the AUV heading align with the flow direction. Even through the AUV may travel a longer distance, it will cut down the required propulsive power. A multi objective cost function was presented in [20]. In this study, path smoothness is modelled as a function of a heading change between two line segments while a clearance is modelled as a function of the smallest distance between line segments to the nearest obstacle. Then, a path distance, smoothness and clearance are combined into a single objective function and effect of each are balanced by using a relative weight rather than a representation of the physical energy consumption as proposed here.

\section{DELPHIN2 AUV}

Delphin2 is an over-actuated AUV [26]. It is equipped with a propeller, four independent control surfaces and four through-body tunnel thrusters (figure 1). With a combination of these actuators, the AUV is over-actuated and is capable of performing both flight-style missions and hovering missions. Extensive studies on the Delphin2 AUV are presented in [27], [28], [29], [30], [31].

Manoeuvres in the horizontal plane can be performed utilizing the rudders, thrusters or a combination of both. It has been shown that using thrusters gives more manoeuvrability at zero or slow forward speeds, i.e. a smaller turning diameter [27]. However, as vehicle speed increases the ambient flow interacts with the thruster jet, decreasing the effective thrust delivered [32], and thus impacting on manoeuvrability. The effect of thruster degradation is included in the simulation and is explained in section VII-C.

In this study, the AUV is assumed to operate at a constant speed and constant depth, i.e. on a horizontal plane. As such, only surge, sway and yaw motion are of interest. In addition, the control surfaces will not be used during transit as for the simplicity. Hence, there are two actuators left to be concerned with. These are the propeller and horizontal thrusters which corresponding to the surge and yaw motion respectively.

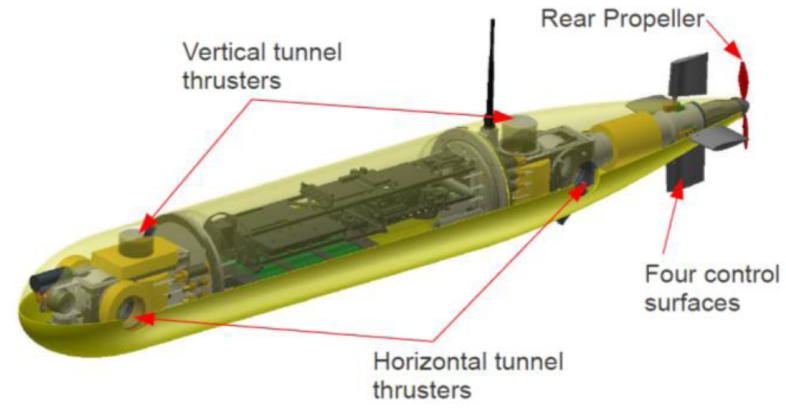

Fig. 1: Delphin2 AUV [26].

\section{ENVIRONMENT REPRESENTATION}

Grid mapping is a technique that decomposes an entire map into a number of non-overlapping cells. Each of these cells holds a binary value corresponding to an occupancy status of occupied or free.

To construct a map, it is required a top-view image of a working space. This can be any kind of picture, including the one that is captured from Google map. Boundaries of the configuration space and obstacles as well as a cell size are needed to be prescribed manually by the user. It is recommended to specify a cell size as a proportion of a vehicle length. Then, the input image is meshed and the occupancy status of each cell is determined accordingly (see figure 2).

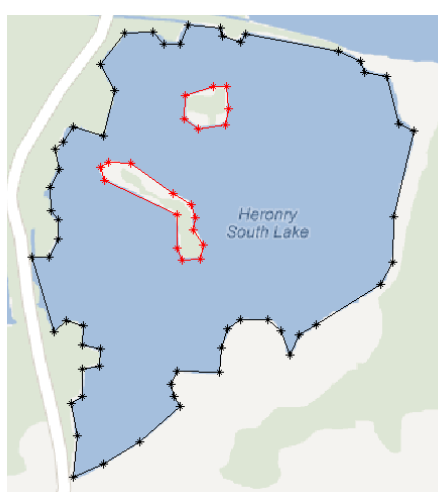

(a) An original map

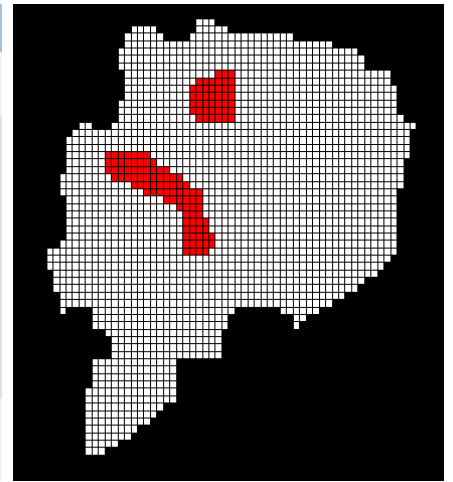

(b) Grid map
Fig. 2: Results of map building and path planning.

\section{GA PATH PLANNING}

The proposed GA technique adopts a non-binary variablelength chromosome coding scheme. This is similar to [20]. The difference is that a corresponding grid index $(i, j)$ is used to represent a chromosome instead of a real $(x, y)$ coordinate. For example, a chromosome of a path in figure 3 is $\{(1,4) ;(3,4) ;(3,3) ;(4,2) ;(4,1)\}$. An initial generation is generated by random walk strategy (see figure 4 ). Then a path refinement - notably node repair and line repair [17] are applied to guarantee all paths in this first generation are feasible. 


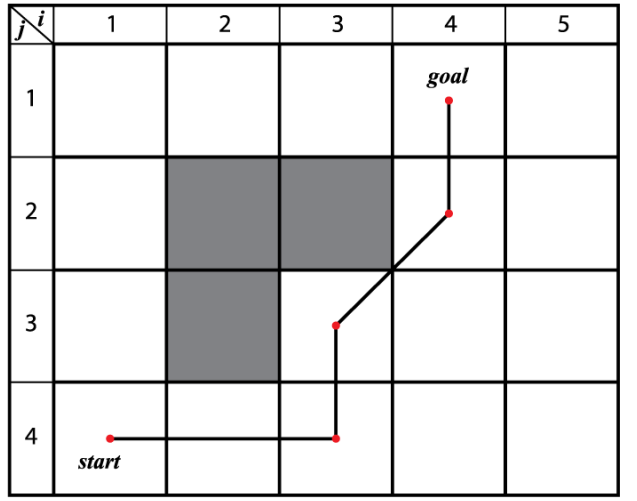

Fig. 3: Example of a valid path.

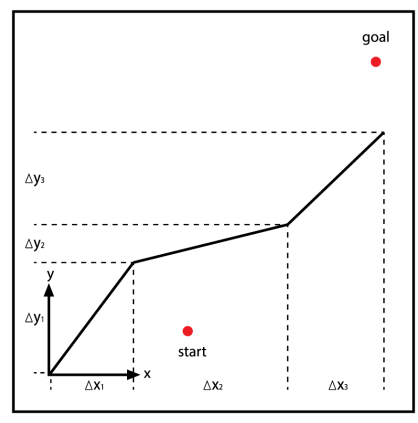

(a) random move

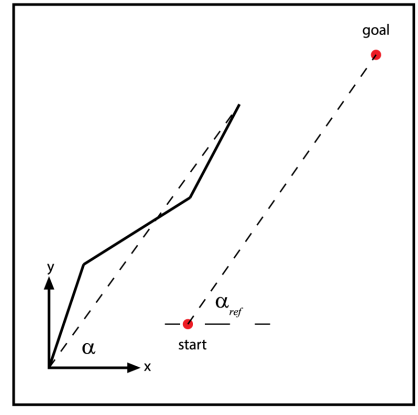

(c) rotating

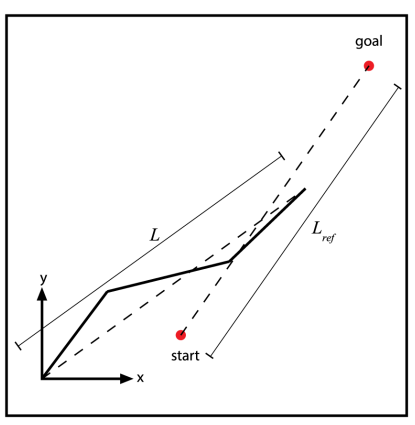

(b) scaling

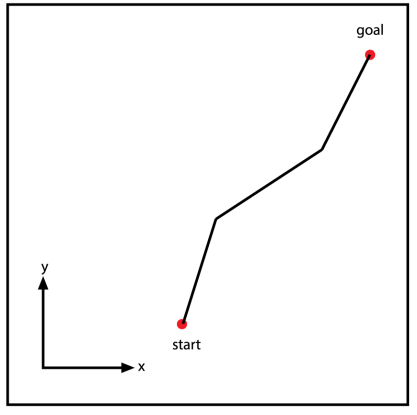

(d) translating
Fig. 4: Four steps of random walk strategy.

A loop of evolutionary process starts by taking in a set of individuals which is referred to as the current generation. Individuals in this generation are then evaluated with a fitness function. The proposed function gives a bias to paths that consume less energy rather than less transit time. This follows by a selection step in which a number of individuals are randomly taken (in bias to its fitness) from the current generation and put into a new set of population called temporary generation. Next, individuals in the temporary generation are transformed by the mean of GA operators, resulting in a next generation. The loop is then repeated until the convergence criteria is achieved (figure 5). In this case, the search will stop once it has reached a maximum number of iterations. The result of this is a set of solutions that contains not only an optimal path but also a number of near-optimal paths that can be used as an alternative path in a case that the environment has changed.

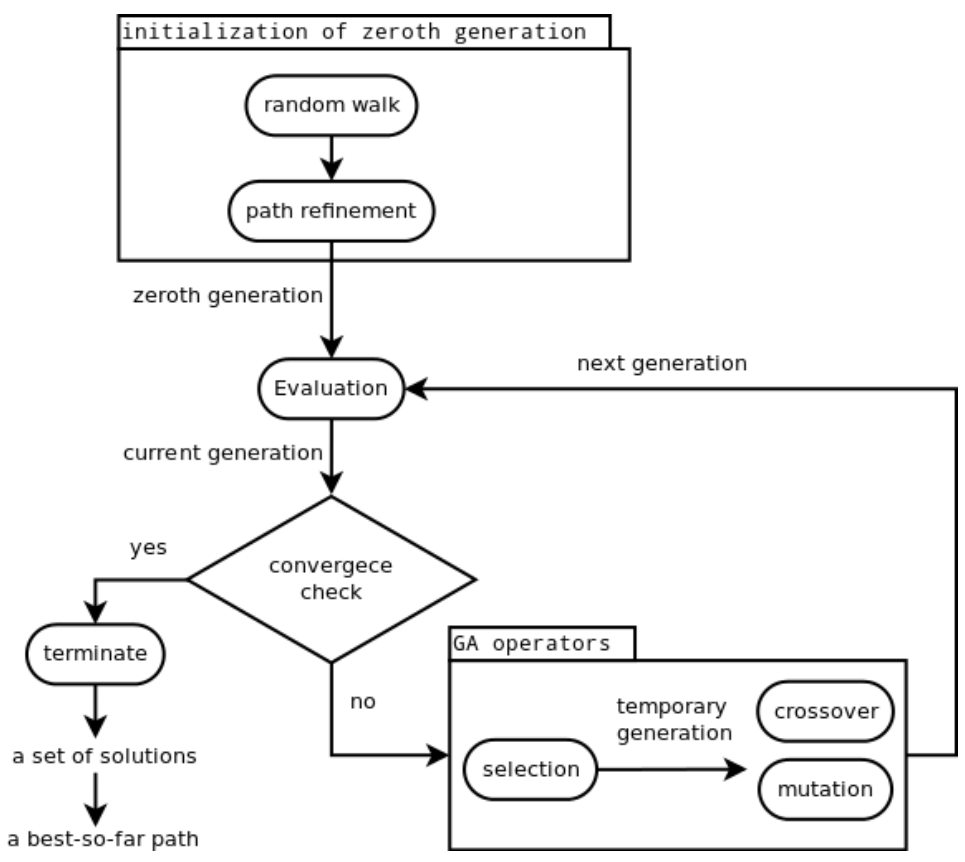

Fig. 5: A diagram presents a loop of Genetic Algorithm.

\section{KEY PARAMETERS OF PATHS}

Assuming that the vehicle is travelling from a waypoint $p_{k-1}$ towards waypoint $p_{k}$. The terms current segment refers to a line segment that connects waypoint $p_{k-1}$ with $p_{k}$. Similarly, the line segment that joins waypoint $p_{k}$ with $p_{k+1}$ is referred as next segment. An extreme length of current segment is denoted as $d$. A manoeuvring distance $d_{m}$ is a distance that the AUV starts to manoeuvre itself to align with the next segment. A remaining distance on the current segment is denoted as propulsion distance $d_{p}$, in which the vehicle aims to move along. The vehicle is assumed to move at a constant forward speed $u$. With this, estimated amount of time $t_{p}$ and $t_{m}$ spent on $d_{p}$ and $d_{m}$ respectively are calculated as follows;

$$
t_{p}=d_{p} / u, \quad t_{m}=d_{m} / u
$$

A relative angle that the next segment makes to the current

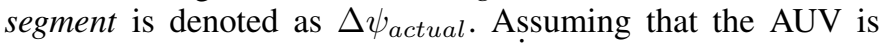
constrained by a saturated yaw rate $\dot{\psi}_{\text {sat }}$, which is a maximum yaw rate that the vehicle can physically perform. This means, there also is a limitation in $\Delta \psi$ that the vehicle can compensate while moving forward. This saturation in heading error is calculated as;

$$
\Delta \psi_{s a t}=\dot{\psi}_{s a t} / t_{m}
$$

If the actual heading error $\Delta \psi_{\text {actual }}$ exceeds this limit, only the $\Delta \psi_{\text {sat }}$ will be compensated while the vehicle is moving along a manoeuvring zone $d_{m}$. The AUV is expected to stop moving forward once it reaches a waypoint $p_{k}$ to compensate a residual heading error;

$$
\Delta \psi_{\text {res }}=\Delta \psi_{\text {actual }}-\Delta \psi_{\text {sat }}
$$


The time spent on manoeuvring with a zero-forward speed is;

$$
t_{m, u 0}=\Delta \psi_{r e s} / \dot{\psi}_{s a t}
$$

All these relevant parameters are illustrated in figure 6 . They are key parameters when evaluating a fitness of the paths in a following section.

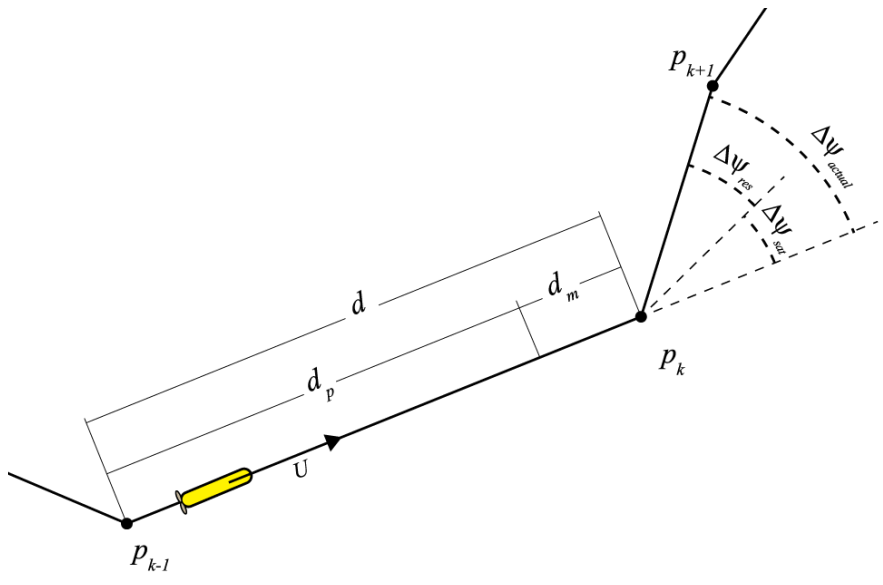

Fig. 6: Path parameters.

\section{FITNESS FUNCTION}

For an AUV to perform long-range missions, endurance is generally the main concern. The cost function presented here is specially derived to minimise an energy consumption basedon the physics of over-actuated AUV, notably Delphin2 AUV. Total energy $\left(E_{\text {total }}\right)$ required by the AUV during a transition can be decomposed into four main components as;

$$
E_{\text {total }}=E_{h}+E_{p}+E_{m}+\text { penalty }
$$

or,

$\int_{0}^{T} P_{\text {total }} \mathrm{d} t=\int_{0}^{T} P_{h} \mathrm{~d} t+\int_{0}^{T} P_{p} \mathrm{~d} t+\int_{0}^{T} P_{m} \mathrm{~d} t+$ penalty

where subscriptions $h, p$ and $m$ refer to hotel, propulsion and manoeuvring respectively. The penalty term is included to differentiate the feasible paths from unfeasible ones.

\section{A. Hotel Load:}

This term represents a total energy consumed by onboard electronics during transit. It is calculated as follows;

$$
E_{h}=\int_{0}^{T} P_{h} \mathrm{~d} t \approx P_{h} \sum_{i=1}^{n_{\text {node }}-1}\left(t_{p}^{[i]}+t_{m}^{[i]}+t_{m, u 0}^{[i]}\right)
$$

where $P_{h}$ is a rate of hotel power consumption (assumed a constant), $t_{p}^{[i]}$ and $t_{m}^{[i]}$ is a time spent on propulsion zone $d_{p}$ and manoeuvring zone $d_{m}$ of $i^{t h}$ path segment, and $t_{m, u 0}^{[i]}$ is time spent on manoeuvring with zero-forward speed.

\section{B. Propulsion Load:}

A propulsive energy consumption rate $\left(P_{p}\right)$ for the AUV to move at a forward speed $u$ is;

$$
P_{p}=\frac{1}{\eta} R_{\text {total }} u
$$

$R_{\text {total }}$ is a total resistance in correspondence with a surge speed $u$ and $\eta$ denotes an efficiency of power transmission. The total resistance is determined as follows;

$$
R_{\text {total }}=\frac{1}{2} \rho c_{d, \nabla} \nabla^{2 / 3} u^{2}
$$

where $\rho$ is water density, $c_{d, \Delta}$ is a volumatic drag coefficient, $\nabla$ is a volume of the vehicle. With this, equation 8 can be rewritten as;

$$
P_{p}=\frac{1}{2 \eta} \rho c_{d, \nabla} \nabla^{2 / 3} u^{3}
$$

This term becomes zero when the vehicle is not moving. In other words, there will be a propulsive power consumption presented only when the AUV is travelling along $d_{p}$ and $d_{m}$. As a consequence, the total propulsive energy is;

$$
E_{p}=\int_{0}^{T} P_{p} \mathrm{~d} t \approx \frac{1}{2 \eta} \rho c_{d, \nabla} \nabla^{2 / 3} u^{3} \sum_{i=1}^{n_{\text {node }}-1}\left(t_{p}^{[i]}+t_{m}^{[i]}\right)
$$

\section{Manoeuvring Load:}

The term manoeuvring load denotes an amount of energy spent by the actuators of the Delphin2 to make itself align with next segment. It is assumed that the yaw motion is performed by utilizing only two horizontal thrusters that are fitted in a front and rear part of the vehicle. Generally, a manoeuvring power $P_{m}$ comsumed by thrusters is varying according to two parameters; thruster setpoint $S P_{\text {thruster }}$ and yaw rate $\dot{\psi}$.

The AUV is subjected to a constraint of manoeuvring distance $d_{m}$ and a maximum yaw rate $\dot{\psi}_{\text {sat }}$ that the vehicle can perform. These two limitations imply that there is a maximum amount of heading error $\left(\Delta \psi_{\text {sat }}\right)$ that the AUV can compensate while moving forward (refer to e.q. 2). If the actual heading error $\Delta \psi_{\text {actual }}$ exceeds this limit, the AUV is expected to stop moving and make itself align with the path first. Once finished, it then continues moving forward.

A yaw rate $\dot{\psi}$ required for the vehicle to manoeuvre in a case of that $\Delta \psi_{\text {actual }} \leq \Delta \psi_{\text {sat }}$ is; 


$$
\begin{aligned}
\dot{\psi} & =\frac{\Delta \psi_{\text {actual }}}{t_{m}} \\
& =\Delta \psi_{\text {actual }} \frac{u}{d_{m}}
\end{aligned}
$$

However, if the $\Delta \psi_{\text {actual }}$ goes beyond a limit of $\Delta \psi_{\text {sat }}$, a yaw rate $\dot{\psi}$ will be saturated and held at $\dot{\psi}_{\text {sat }}$. A required yaw rate conditioned to an actual heading error is expressed as a piecewise function as follows;

$$
\dot{\psi}= \begin{cases}\dot{\psi}_{\text {sat }} & \text {;if } \Delta \psi_{\text {actual }}>\Delta \psi_{\text {sat }} \\ \Delta \psi_{\text {actual }} \frac{u}{d_{m}} & ; \text { otherwise }\end{cases}
$$

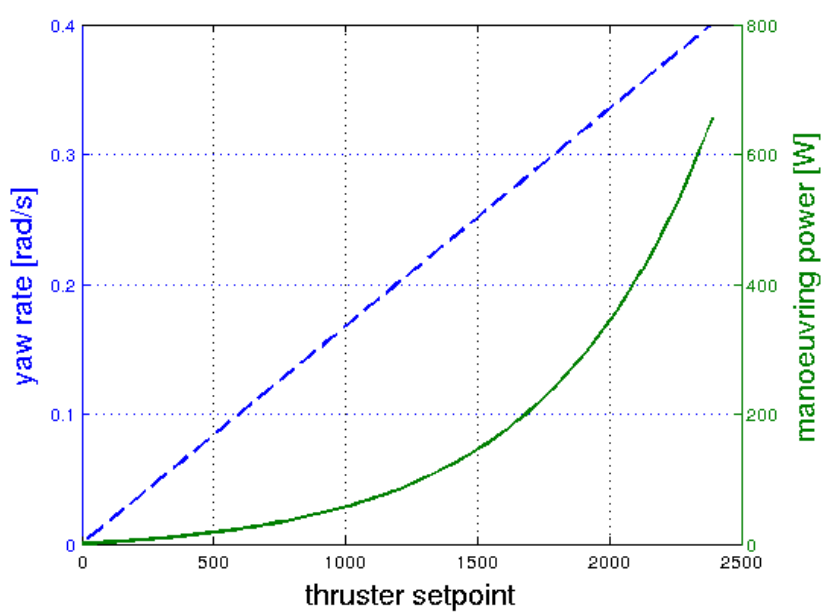

Fig. 7: A relationship between $S P_{\text {thruster }}$ and $\dot{\psi}$ is denoted by a blue dash line whereas a relationship between $S P_{\text {thruster }}$ and $P_{m}$ is denoted by a green solid line.

By means of experiment, one can find a relationship between yaw rate $\dot{\psi}$, thruster setpoint $S P_{\text {thruster }}$ and a power consumption due to manoeuvring $P_{m}$ at a fixed speed $u$. This will look somewhat like what shown in figure 7 . With a required yaw rate $\dot{\psi}$, a corresponding thruster setpoint $S P_{\text {thruster }}$ is determined, and, in turn, a rate of manoeuvring power consumption $P_{m}(\dot{\psi}, u)$ is obtained. The manoeuvring energy for the whole path is determined as;

$$
E_{m}=\int_{0}^{T} P_{m}(\dot{\psi}, u) \mathrm{d} t \approx \sum_{i=1}^{n_{\text {node }}-1} P_{m}(\dot{\psi}[i], u)\left(t_{m}^{[i]}+t_{m, u 0}^{[i]}\right)
$$

It is pointed out in [32] that there will be a drop in thruster performance which happens when the vehicle is undergoing forward motion. This is because the effect of a jet emitted from the thruster exit is dominated by the ambient flow (surge speed in this case). As such, it is common to express the thruster degradation as a function of a speed ratio which is a ratio of a surge speed $(u)$ to a thruster jet-speed $\left(u_{j}\right)$, see figure 8 . It is possible that thrusting force will drop to below $20 \%$ of the force in a static condition. In future, experiments will be used to establish a realistic relationship between thruster power,

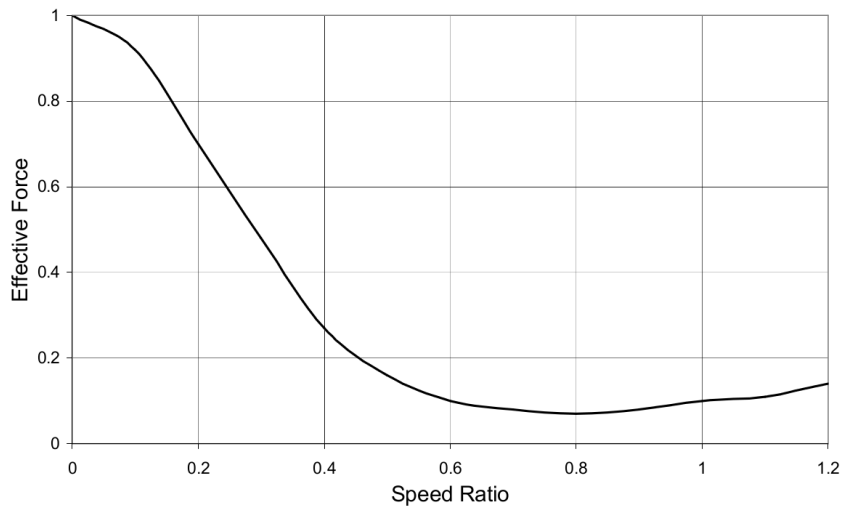

Fig. 8: A variation in effective thrusting force with a speed ratio [32].

forward speed and yaw rate. At this time, thruster degradation has been modelled by assuming that the propulsion power to achieve a specific yaw rate at a forward speed of $0.6 \mathrm{~m} / \mathrm{s}$ is 5 times greater than of at the zero forward speed.

\section{Penalty:}

Role of this term is to differentiate unfeasible paths from the feasible ones by adding an enormous amount of extra energy consumption to the path if a collision is detected. To make this penalty term adaptive to arbitrary cases, it is defined as a proportion of the energy consumption when the AUV transits on a direct path from start point to goal with a constant forward speed $u$. As this virtual path is a straight line, there will be no manoeuvring energy term presented. The penalty term is as follows;

$$
\text { penalty }=\sigma N\left(P_{h}+P_{p}\right) T_{0}
$$

where $P_{h}$ and $P_{p}$ are determined as explained earlier, $N$ is a number of times that the path collides with obstacles, and $\sigma$ is a parameter to adjust the relative weight of the penalty term. $T_{0}$ is a time the vehicle spent on the virtual path. This is calculated using a direct distance from start point to goal $D_{0}$ as;

$$
T_{0}=\frac{D_{0}}{u}
$$

\section{E. Fitness:}

By substituting equation 7, 11, 14 and 15 into equation 5, one can evaluate a fitness of the path. The path that has smallest $E_{\text {total }}$ is the best path. However, GA technique requires to have the highest score corresponds to the best path. As a consequence, fitness of path must be redefined as;

$$
\text { fitness }=\frac{1}{E_{\text {total }}}
$$




\section{Simulation SETUP}

There were three test cases corresponding to three types of maps. First case was a simple and realistic environment of a Heronry South Lake with an actual map size of $544 \times 581$ metres. Second case was a labyrinth like structure that contains a number of concave-shaped obstacles. Third case was a split-ellipse map that has a shortest path pass through the middle. Map size of both second and third case were equivalent to $550 \times 550$ metres. These maps were meshed with a square grid with its width is twice as big as the AUV length.

In the simulation, a surge speed $u=0.6 \mathrm{~m} / \mathrm{s}$ and a maximum yaw rate $\dot{\psi}_{\text {sat }}=0.3 \mathrm{rad} / \mathrm{s}$ were used. These values were estimated according to actual dynamics of the Delphin2 AUV. A manoeuvring distance $d_{m}$ was also specified as a proportion of the vehicle length, and in this case it was two times the length. A scale for the penalty term is $\sigma=1000$.

GA parameters used in the simulation were population size $=200$, number of generation $=80$, crossover rate $=0.8$ and mutation rate $=0.3$.

\section{RESUlTS AND Discussion}

Path planning simulation was written in MatLab. In each test case, GA with an improved cost function was employed to search for an optimal energy path, while a conventional GA and $\mathrm{A}^{*}$ technique were employed to search for an optimal distance path. Results from the conventional GA and $\mathrm{A}^{*}$ approach were post-processed further to determine an amount of energy required to navigate along those paths. The solutions of $\mathrm{A}^{*}$ were used as a benchmark to justify a performance of both versions of GA.

It is noted that, all these results were non-dimensionalized by a virtual straight line path leading from start to goal location. For example, a path distance $(d)$ of each test case was divided by a distance of a direct path leading from same start to same goal location. This results in a non-dimensionalized distance $d^{\prime}$. For the same purpose, all energy terms were divided by a hotel load of when the vehicle is travelling on the direct path. A non-dimensional term of hotel, propulsion, manoeuvring and total energy are denoted as $E_{h}^{\prime}, E_{p}^{\prime}, E_{m}^{\prime}$ and $E_{\text {total }}^{\prime}$ respectively. Beside, a \%change presented in the tables is a change in outcomes of GA approaches relative to the ones of the $A^{*}$. The results of three test cases are presented below.

The results from three cases clearly show that a conventional GA (aimed at optimal distance paths) yields slightly shorter paths which have a substantial reduction in a number of turns compared with ones of $\mathrm{A}^{*}$ approach. A result of this is a decrease in total energy consumption. It is because the GA is not constrained by 8-connectivity links, hence, avoid making unnecessary turns in which wasting energy.

Comparing two versions of GA, in case 1 and 2, outcomes of both versions are almost identical and again slightly shorter and much smoother compared with $A^{*}$ (see figure 9 and
10). What interesting is in case 3 in which their results are entirely different (see figure 11). The conventional GA suggests to use an inner path which is a best strategy in terms of travelling distance. In contrast, the improved GA suggests an outer path which is $10 \%$ longer in distance compared with $\mathrm{A}^{*}$. However, this come up with a further reduction in a total energy consumption as much less manoeuvring effort is required.

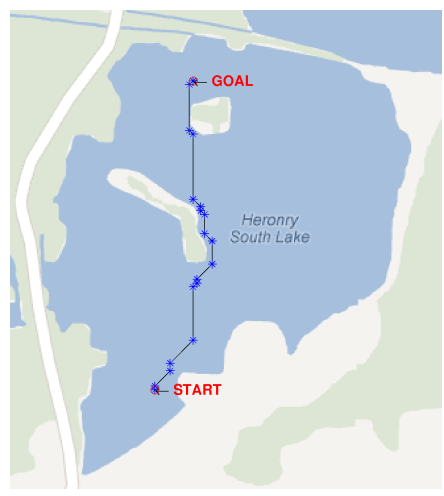

(a) $\mathrm{A}^{*}$

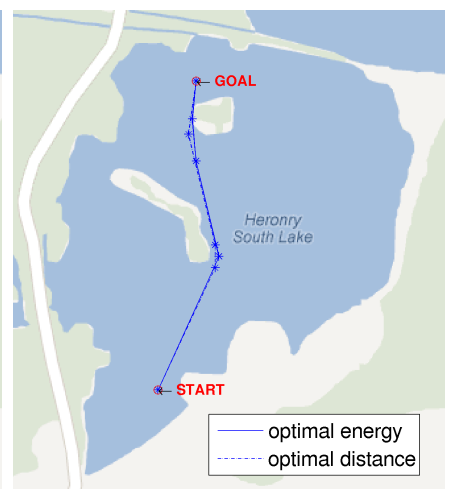

(b) GA
Fig. 9: Case 1 - Heronry souhth lake.

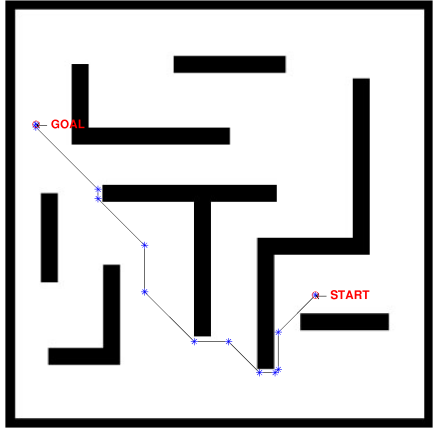

(a) $\mathrm{A}^{*}$

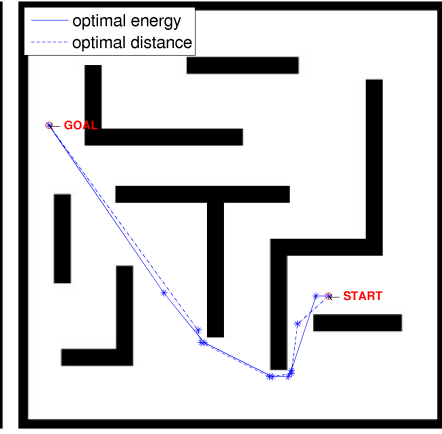

(b) GA
Fig. 10: Case 2 - Labyrinth.

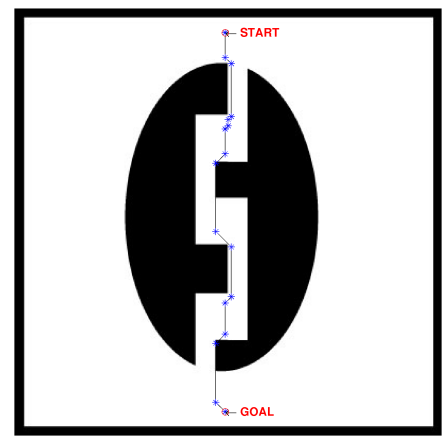

(a) $\mathrm{A}^{*}$

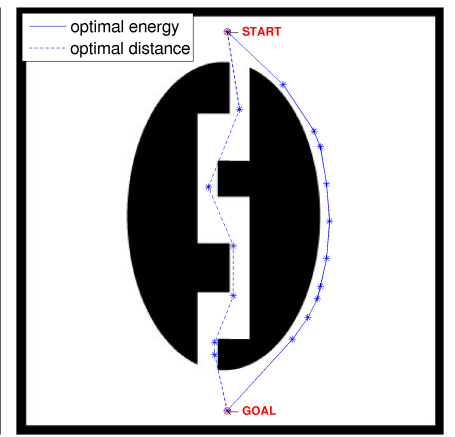

(b) GA
Fig. 11: Case 3 - Split ellipse. 
Even though GA approaches yield shorter and smoother outcomes than $\mathrm{A}^{*}$, they take a substantial more amount of computing time to find a solution. In the experiment, $A^{*}$ takes less than a second while both versions of GA approaches take almost 10 seconds. Furthermore, GA is a stochastic search that is sensitive to a parameter tuning issue. For example, if a number of generation is reduced, it may not have enough time to converge to a global solution. $\mathrm{A}^{*}$ which is a deterministic search does not have this parameter tuning problem. With these drawbacks, GA techniques may only be suitable for off-line path planning problem that the path is planned in advance and can be approved by the operator.

\begin{tabular}{|l|c|c|r|r|r|}
\hline & $\mathrm{A}^{*}$ & $\mathrm{GA}_{\text {dis }}$ & \% change & $\mathrm{GA}_{\text {ener }}$ & \% change \\
\hline$d^{\prime}$ & 1.104 & 1.045 & -5.323 & 1.045 & -5.353 \\
\hline$E_{h}^{\prime}$ & 1.351 & 1.055 & -21.909 & 1.056 & -21.784 \\
\hline$E_{p}^{\prime}$ & 1.598 & 1.513 & -5.323 & 1.512 & -5.353 \\
\hline$E_{m}^{\prime}$ & 4.663 & 0.368 & -92.106 & 0.295 & -93.674 \\
\hline$E_{\text {total }}^{\prime}$ & 7.612 & 2.936 & -61.433 & 2.864 & -62.377 \\
\hline time (sec) & 0.513 & 8.021 & - & 8.227 & - \\
\hline
\end{tabular}

TABLE I: Case 1 - Heronry souhth lake.

\begin{tabular}{|l|c|c|r|r|r|}
\hline & $\mathrm{A}^{*}$ & $\mathrm{GA}_{\text {dis }}$ & \% change & $\mathrm{GA}_{\text {ener }}$ & \% change \\
\hline$d^{\prime}$ & 1.438 & 1.393 & -3.082 & 1.396 & -2.931 \\
\hline$E_{h}^{\prime}$ & 1.561 & 1.448 & -7.224 & 1.443 & -7.594 \\
\hline$E_{p}^{\prime}$ & 2.081 & 2.017 & -3.082 & 2.020 & -2.931 \\
\hline$E_{m}^{\prime}$ & 2.335 & 1.046 & -55.209 & 0.897 & -61.578 \\
\hline$E_{\text {total }}^{\prime}$ & 5.977 & 4.511 & -24.527 & 4.360 & -27.059 \\
\hline time (sec) & 0.820 & 9.063 & - & 9.796 & - \\
\hline
\end{tabular}

TABLE II: Case 2 - Labyrinth.

\begin{tabular}{|l|c|r|r|r|r|}
\hline & $\mathrm{A}^{*}$ & $\mathrm{GA}_{\text {dis }}$ & \% change & $\mathrm{GA}_{\text {ener }}$ & \% change \\
\hline$d^{\prime}$ & 1.068 & 1.045 & -2.172 & 1.182 & 10.695 \\
\hline$E_{h}^{\prime}$ & 1.213 & 1.068 & -12.002 & 1.182 & -2.574 \\
\hline$E_{p}^{\prime}$ & 1.546 & 1.512 & -2.172 & 1.711 & 10.695 \\
\hline$E_{m}^{\prime}$ & 2.753 & 0.623 & -77.380 & 0.233 & -91.527 \\
\hline$E_{\text {total }}^{\prime}$ & 5.512 & 3.202 & -41.897 & 3.126 & -43.279 \\
\hline time (sec) & 0.489 & 8.046 & - & 8.176 & - \\
\hline
\end{tabular}

TABLE III: Case 3 - Split ellipse.

\section{Conclusion And Future Work}

In this paper an improved cost function for grid-based genetic algorithm (GA) path planning was presented. This function consists of relevant indicators that are turned into a common unit of energy so they are comparable. Precisely, a travelling distance is turned to a hotel and propulsion load while a smoothness is turned to a manoeuvring load. Then, it seeks for a good compromise between a travelling distance and a smoothness. A simulation of this was implemented in MatLab. The results were compared with other approaches, notably conventional GA and $\mathrm{A}^{*}$, that seek an optimal distance path. The outcomes clearly show that optimal solutions from improved GA consume less energy than the ones obtained from other two approaches. More importantly, the solution of both versions of GA contain less number of sharp turns than $A^{*}$ as there is no movement constrained applied. So, the path looks more realistic and requires less manoeuvring effort.
A major drawback of GA techniques is that they are sensitive to a parameter tuning issue. If the algorithm was not tuned properly, it may output a sub-optimal solution. This is opposite to the $\mathrm{A}^{*}$ approach which is a deterministic search that guarantees an optimal solution if the solution does exist. Beside, a time to convergence increases substantially. Due to this, the proposed GA technique may not yet suitable for an on-line path planning.

A future work would be focusing on how to reduce a convergence time and increase a reliability of the GA approach. This could make GA compatible with an on-line path planning problem. Beside, in this work, a thruster degradation with forward speed is assumed a constant which is not physically valid. This must be altered by a proper model of thruster in which a degradation is varying with a thruster setpoint and vehicle speed. In later work, variable forward speed will be used and the effect of the use of control surfaces on manoeuvrability will be included. Experiment will be proposed and validate with the simulation result. In addition, map making process would be made automatically, e.g. using an image processing technique to specify a boundary of working space and obstacles.

\section{REFERENCES}

[1] P. Jantapremjit and P. A. Wilson, "Guidance-Control Based Path Following for Homing and Docking using an Autonomous Underwater Vehicle," OCEANS 2008 - MTS/IEEE Kobe Techno-Ocean, no. 1, pp. 1-6, Apr. 2008.

[2] Y. Koren and J. Borenstein, "Potential field methods and their inherent limitations for mobile robot navigation," in Proceedings. 1991 IEEE International Conference on Robotics and Automation. IEEE Comput. Soc. Press, 1991, pp. 1398-1404.

[3] X. Yun and K. Tan, "A wall-following method for escaping local minima in potential field based motion planning," in 1997 8th International Conference on Advanced Robotics. Proceedings. ICAR'97. IEEE, 1997, pp. 421-426.

[4] L. Chengqing, M. Ang, H. Krishnan, and L. S. Yong, "Virtual obstacle concept for local-minimum-recovery in potential-field based navigation," in Proceedings 2000 ICRA. Millennium Conference. IEEE International Conference on Robotics and Automation. Symposia Proceedings (Cat. No.00CH37065), vol. 2, no. April. IEEE, 2000, pp. 983-988.

[5] D. Fu-guang and J. Peng, "AUV local path planning based on virtual potential field," in IEEE International Conference Mechatronics and Automation, 2005, vol. 4, no. July. IEEE, 2005, pp. 1711-1716.

[6] M. Mabrouk and C. McInnes, "Solving the potential field local minimum problem using internal agent states," Robotics and Autonomous Systems, vol. 56, no. 12, pp. 1050-1060, Dec. 2008.

[7] J. Hu, W. gen Wan, and X. Yu, "A pathfinding algorithm in real-time strategy game based on Unity3D," in 2012 International Conference on Audio, Language and Image Processing. IEEE, Jul. 2012, pp. 11591162.

[8] M. Likhachev, D. Ferguson, and G. Gordon, "Anytime Dynamic A*: An Anytime, Replanning Algorithm." ICAPS, 2005.

[9] S. Koenig and M. Likhachev, "Fast replanning for navigation in unknown terrain," Robotics, IEEE Transactions on, vol. XX, 2005.

[10] D. Ferguson and A. Stentz, "Using interpolation to improve path planning: The Field D* algorithm," Journal of Field Robotics, vol. 23, no. 2, pp. 79-101, 2006.

[11] V. Ganapathy, S. Yun, and T. Chien, "Enhanced D* Lite Algorithm for Autonomous Mobile Robot," International Journal of Applied, vol. 1, no. 1, pp. 58-73, 2011.

[12] D. Sharma and S. K. Dubey, "Anytime A* Algorithm-An Extension to A* Algorithm," vol. 4, no. 1, pp. 1-4, 2013. 
[13] M. Barbehenn, "A note on the complexity of Dijkstra's algorithm for graphs with weighted vertices," Computers, IEEE Transactions on, vol. 47, no. 2, p. 106062, 1998.

[14] L. D. Davis, "Handbook Of Genetic Algorithms." Van Nostrand Reinhold, 1991.

[15] Z. Michalewicz, Genetic Algorithms + Data Structures = Evolution Programs (2Nd, Extended Ed.). New York, NY, USA: Springer-Verlag New York, Inc., 1994.

[16] K. Sugihara and J. Smith, "A genetic algorithm for 3-d path planning of a mobile robot," Department of Information and Computer Science, University of Hawaii at Manoa, Tech. Report, 1996.

[17] S. Yang, "A knowledge based genetic algorithm for path planning of a mobile robot," in IEEE International Conference on Robotics and Automation, 2004. Proceedings. ICRA '04. 2004, vol. 5, no. 26 April-1 May 2004. IEEE, 2004, pp. 4350-4355 Vol.5.

[18] Q. Li, W. Zhang, Y. Yin, Z. Wang, and G. Liu, "An Improved Genetic Algorithm of Optimum Path Planning for Mobile Robots," Sixth International Conference on Intelligent Systems Design and Applications, vol. 2, pp. 637-642, Oct. 2006.

[19] Z. Yao and L. Ma, "A Static Environment-Based Path Planning Method by Using Genetic Algorithm," 2010 International Conference on Computing, Control and Industrial Engineering, pp. 405-407, 2010.

[20] A. Elshamli, H. Abdullah, and S. Areibi, "Genetic algorithm for dynamic path planning," in Canadian Conference on Electrical and Computer Engineering 2004 (IEEE Cat. No.04CH37513). IEEE, 2004, pp. $677-680$.

[21] K. Sugihara and J. Yuh, "GA-based motion planning for underwater robotic vehicles," Proceedings of the 10th International Symposium on Unmanned Untethered Submersible Technology, Autonomous Undersea Systems Institute, 1997.

[22] K. Sugihara and J. Smith, "Genetic algorithms for adaptive motion planning of an autonomous mobile robot," in Proceedings 1997 IEEE International Symposium on Computational Intelligence in Robotics and Automation CIRA'97. 'Towards New Computational Principles for Robotics and Automation'. IEEE Comput. Soc. Press, 1997, pp. 138143.

[23] J. Tu and S. X. Yang, "Genetic algorithm based path planning for a mobile robot," Robotics and Automation, 2003. Proceedings. ICRA'03. IEEE International Conference on, vol. 1, no. 0, pp. 1221-1226, 2003.

[24] A. Alvarez, A. Caiti, and R. Onken, "Evolutionary Path Planning for Autonomous Underwater Vehicles in a Variable Ocean," IEEE Journal of Oceanic Engineering, vol. 29, no. 2, pp. 418-429, Apr. 2004.

[25] A. Lammas and K. Sammut, "Optimal path planning based on annular space decomposition for AUVs operating in a variable environment," 2012 IEEE/OES Autonomous Underwater Vehicles (AUV), pp. 1-9, Sep. 2012.

[26] A.B. Phillips et. al., "Delphin2: An over actuated autonomous underwater vehicle for manoeuvring research," Transactions of the Royal Institution of Naval Architects, Part A Engineering, pp. 1-11, 2013.

[27] L.V. Steenson, A.B. Phillips, and M.E. Furlong, "Maneuvering of an over-actuated autonomous underwater vehicle using both through-body tunnel thrusters and control surfaces," 2011.

[28] L.V. Steenson, A.B. Phillips, M. Furlong, E. Rogers, and S.R. Turnock, "The performance of vertical tunnel thrusters on an autonomous underwater vehicle operating near the free surface in waves," no. June, 2011.

[29] L.V. Steenson, A.B. Phillips, E. Rogers, M. Furlong, and S.R. Turnock, "Preliminary results of a hover capable AUV attempting transitional flight," Unmanned Untethered Submersible Technology (UUST), pp. 19, 2011.

[30] L.V. Steenson, "Experimentally Verified Model Predictive Control of a Hover-Capable AUV," Ph.D. dissertation, University of Southampton, 2013.

[31] L.V. Steenson, S.R. Turnock, A.B. Phillips, C. Harris, M.E. Furlong, E. Rogers, L. Wang, K. Bodles, and D.W. Evans, "Model predictive control of a hybrid autonomous underwater vehicle with experimental verification," Proceedings of the Institution of Mechanical Engineers, Part M: Journal of Engineering for the Maritime Environment, vol. 228, no. 2, pp. 166-179, Feb. 2014.
[32] A. Palmer, G. Hearn, and P. Stevenson, "Modelling tunnel thrusters for autonomous underwater vehicles," in Navigation, Guidance and Control of Underwater Vehicles (NGCUV'08). International Federation of Automatic Control (IFAC). 